with the experimental results for 135,480 , and 812 volt electrons by using a similar method.

In a co-ordinate system in which one atom is initially at rest, the number of atoms scattered between the angles $\Theta$ and $\Theta+d \Theta$ will be of the form $I(\Theta) \sin 2 \Theta d \Theta$. The function $I(\Theta)$ obtained for the helium atom collisions is illustrated in Fig. 1, showing the existence of marked diffraction effects.

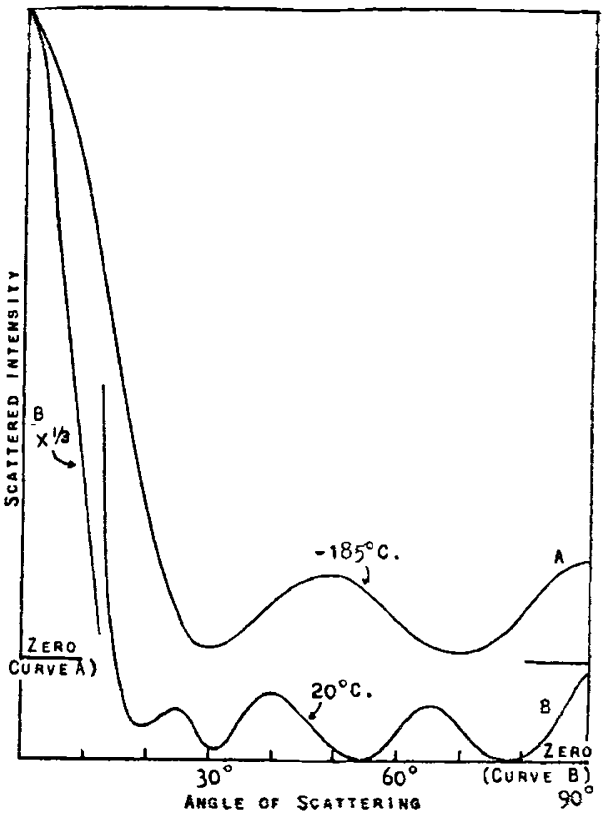

Fla. 1.-Angular distribution of function $I(8)$ for helium atom scattered by helium atoms with relative velocities correspondin scattered by helium atoms with relative

Allowance has been made for the identity of the helium atoms. ${ }^{7}$ The collision diameter is very nearly the same for both relative velocities, being $2 \cdot 4 \mathrm{~A}$.

Unfortunately, direct experimental verification of these curves is difficult, owing to the necessity of obtaining monochromatic molecular beams. However, the effect should be apparent in the viscosity and other transport phenomena in helium. It is hoped to investigate this later. H. S. W. Massey.

Trinity College, Cambridge, July 25.

'Phys. Rev., 37, 682; 1931.

Z. Phys., 45, 307; 1927

3 Proc. Lond. Math. Soc. (2), 23, 428 ; 1924.

Vide Mott, Proc. Cam. Phil. Soc. 25, 304; 1928

5 Arnot, Proc. Roy. Soc., A, 130, 655; 1931.

6 Naturwiss., 30, $561 ; 1932$.

Ilott, Proc. Roy. Soc., A, 126, 259; 1930.

\section{The Velocity of Light}

IN articles in Nature and elsewhere, M. E. J. Gheury de Bray has proposed and defended the suggestion that the velocity of light may be a decreasing function of time, varying at present by about $4 \mathrm{~km}$. a second a year. He bases this idea on an examination of the published results of measurements by a number of experimenters over a period of several decades, and concludes that all the results are in good agreement with his hypothesis.

I have obtained experimental proof that no such variability of velocity exists; the work was an offshoot of an investigation carried out at the California Institute of Technology to test whether time conforms to the requirements of the theory of relativity. An interference arrangement was employed in which the re- tardation of the interfering beams was $318 \mathrm{~mm}$. or 582,000 wave-lengths of the mercury line used as the source. It proved feasible and easy to measure shifts in the interference pattern with a probable error of a thousandth of a fringe. This accuracy was attained by photographing the interference rings and comparing each photograph directly with a nearly identical one used as standard of reference for the whole series. The interference apparatus was built almost entirely of fused quartz and kept in a vacuum at an accurately controlled temperature, while the source, which was an electrodeless high-frequency discharge in unsaturated mercury vapour, was so contrived as to be free from spurious frequency shifts such as might result from Doppler effects, pressure variations, and so forth. The apparatus and procedure are described in detail in a paper entitled "Experimental Establishment of the Relativity of Time", by myself and E. M. Thorndike, to appear in the Physical Review.

The number of waves retardation in any interference arrangement is

$$
n=\frac{\nu \Delta s}{c}
$$

where $v$ is the frequency and $c$ is the velocity of light, while $\Delta s$ is the path-difference. If $\nu$ and $\Delta s$ are supposed constant while $c$ is a function of time, the rate of variation of $n$ is thus

$$
\frac{d n}{d t}=-\frac{\nu \Delta s}{c^{2}} d c
$$

$\Delta s=318 \mathrm{~cm} ., c=3 \times 10^{10}, \nu=6 \times 10^{14}$, and according to de Bray's hypothesis $\frac{d c}{d t}=-4 \mathrm{~km}$. per sec. per year $=-1100 \mathrm{~cm}$. per sec. per day. Hence we should find

$$
\frac{d n}{d t}=0 \cdot 023 \text { fringe per day. }
$$

Three series of data were taken over periods varying from 8 days to a month, and the computed daily rates of change, expressed in thousandths of a fringe a day, are $0.050 \pm 0.020,0.007+0.013$, and $-0.015 \pm 0.021$. Their mean is $0.014 \pm 0.011$. This value stands in the ratio $6 \times 10^{-4}$ to the amount required by the hypothesis of variable velocity, and of course definitely disposes of the possibility of it, unless the frequency itself is supposed to vary correspondingly.

University of Washington, Seattle, July 5.

\section{Metabolic Rate and Habitat}

WHILE innumerable adaptations in structure and habits of animals have been described, we have as yet little knowledge of the physiological causes and effects of distribution and habitat.

As regards metabolism, it is known that trout and mackerel, fishes confined to well-oxygenated waters, have hæmoglobins with a relatively small affinity for oxygen.1, 2 Their blood cedes its oxygen readily to the tissues, permitting of an active life, but in water deficient in oxygen these fishes suffer. Carp, on the contrary, lead a sluggish life. Their hæmoglobin parts reluctantiy with its oxygen, but it can capture the gas from poorly aerated waters and so enable the fish to survive. Trout, in effect, consume more oxygen than carp. ${ }^{3}, 4$ The squid and the king crab offer a like contrast of blood with hæmocyanins. ${ }^{5}$ Again, the oxygen affinity of respiratory pigments varies with temperature, and this circumstance may limit poikilothermal animals to different latitudes. ${ }^{6}$

Some marine invertebrates can live in brackish water, and it has been shown that these animals consume more oxygen in the fresher waters than in the 